Results: Of the 3488 participants, $12.5 \%$ (436/3488) met criteria for depression. Of the population, $8.9 \%$ had a mild depression and 3.9\% met criteria for a moderate to severe depression. Depression was statistically significantly higher among women, adults living alone, those separated or divorced, and those unemployed, working part, casually employed or a student. Individuals with a degree or higher level of education, and those earning above $\$ 40 \quad 000$ were significantly less likely to have depression.

Conclusions: The demographics for those with depression are consistent with previously published studies of depression in the community. However, the rates of depression were higher than that seen in other South Australian, Australian and international studies. The possible reasons for this will be discussed.

\section{Use of the web in the self-management of bipolar disorder}

\section{J Proudfoot, G Parker, S Grdovic, L Greenfield, M Hyett}

The Black Dog Institute, Sydney, Australia

Background: Poor treatment adherence in people with bipolar disorder is common, and $40 \%$ relapse within a year. Education and support assist sufferers to selfmanage their condition and reduce hospitalizations, but the challenge lies in providing them in a convenient, private and nonstigmatizing manner.

Computer-based programs are acceptable to patients and efficacious, but debate exists about whether additional support is needed to maximize effects. This paper reports a study evaluating a web-based education program for people with bipolar disorder.

Methods: Developed at the Black Dog Institute by health professionals, community representatives and consumers, the Bipolar Education Program aims to help individuals and their families better understand the condition, recognize early warning signs, reduce triggers, develop self-management skills and enhance treatment compliance. Modules involve audiovisual presentations, questions and answers, advice from wellknown people with bipolar disorder and printouts.

A randomized controlled trial (RCT) is being conducted, comparing three versions of the program (on its own, with email support from an informed person, with informed support plus assistance to implement a 'staywell' plan) to minimal online education. Measures are being taken at pre-, post-, 3 and 6 months follow-up of clinical and psychosocial outcomes, and costs.

Results: Since its May 2006 launch, the Web site has recorded approximately 2000 visits per month. About $75 \%$ of first-time visitors have been women, 55\% aged 31-50, 44\% with bipolar disorder (predominantly $\leq 12$ months since diagnosis), $28 \%$ carers/family/friends and $18 \%$ health professionals. The RCT is about to commence, data from which will be presented at the conference.

\section{Keeping Care Complete: results from the first international schizophrenia and bipolar disorder family caregivers survey}

\section{R Ramjan \\ Schizophrenia Fellowship of NSW Inc., Sydney, Australia}

Background: The international shift toward community care and the deinstitutionalization of people with mental illness has seen much of the responsibility for day-to-day care transferred to family members. This responsibility can be associated with profound psychosocial, physical and financial impact. Keeping Care Complete is the first large, international survey of carers of people with schizophrenia or bipolar disorder.

Method: About 100 Australian carers were enrolled in an eight-country survey of 982 carers of people with schizophrenia or bipolar disorder. Participants were asked a series of questions regarding treatment, treatment discontinuation, relapse, satisfaction with treatment and carer burden. This was done by means of a 30-min telephone interview.

Results: Australian carers are much involved in their family member's treatment: $80 \%$ of those surveyed reported being the primary carer, $42 \%$ spent more than $20 \mathrm{~h}$ /week caring for their relative and 19\% were responsible for administering medications. For 97\% of carers, family support was the key factor, as well as medication, for keeping their family member well and $70 \%$ of the participants were frustrated by the low expectations for long-term improvement set by health care professionals.

Conclusion: This survey provides significant insights into the experiences of carers of people with mental illness and helps quantify the impact of this responsibility on carers in terms of their physical, mental and financial well-being.

\section{A randomized controlled trial to assess the efficacy of omega-3 fatty acids as a treatment for depression during the perinatal period}

\section{A-M Rees, G Parker, M-P Austin}

The Black Dog Institute, Sydney, Australia 
Background: Epidemiological studies assessing rates of postnatal depression in relation to levels of fish consumption and reduced levels of omega- 3 in the perinatal period indicate a possible link between lowered long-chain omega- 3 fatty acids and perinatal depression.

Aim: To assess whether omega-3 fatty acid treatment is superior to placebo in the treatment of perinatal depression.

Methods: In a double-blind, randomized, placebocontrolled treatment trial, we recruited women from third trimester to 6 months postpartum with a diagnosis of major depression. Treatment was for 6 weeks with either $6 \mathrm{~g}$ of fish oil or placebo. Weekly followup was carried out by a psychiatrist and depression rating scales recorded. Fatty acid analysis was conducted on blood samples collected at baseline and posttreatment trial.

Results: There was significant improvement in depression scores for the treatment group during the trial. However, a repeated-measures ANOVA showed no statistically significant difference between treatment and placebo groups using intention-to-treat analysis. Trend for efficacy differentiation were noted when nonresponders and rapid remitters were excluded.

Conclusions: These results are likely to be affected by a strong placebo response, which is a common problem in clinical trials for the treatment of depression. Further study is needed in this area because treatment options are limited for perinatal depression.

\section{Understanding how young people cope with distress: the development of a mobile phone momentary sampling program (Mobile_TYPE)}

\section{S Reid', G Patton', L Sanci'2, S Kauer'}

'Murdoch Childrens Research Institute; and 2Department of General Practice, University of Melbourne, Melbourne, Australia

Background: Young people report that they typically respond to distress by talking to their friends, thinking and distracting themselves. By using retrospective questionnaires, research has examined what young people say but not what they actually do when distressed and what the impact of these responses are prospectively. The aim of this study was to design a mobile phone momentary sampling program that prospectively tracks the everyday mood, stresses and coping strategies of young people as they happen.

Design: A momentary sampling program was designed and translated into java-based language for mobile phones. The program ran for 7 days, administering four random samples per day. Participants were prompted to report current activity, companions, mood, response to low mood, recent stressful experiences, and alcohol and cannabis use. The program was reviewed by 11 high school students in focus groups, and 18 students completed 7 days of mobile monitoring.

Results: Engagement with the Mobile_TYPE program was high with a majority of students $(82 \%)$ completing at least $75 \%$ of the entries. The most common responses to feeling distressed were do nothing (14.6\%), eating $(11.4 \%)$, sleeping (10.8\%) and studying (7.5\%). About $88 \%$ of active responses to distress lead to a later report of feeling good or better. The responses associated with a later report of not feeling better were worrying, doing nothing, crying, relaxing, eating, studying, pretending to be okay and shopping.

Conclusion: The Mobile_TYPE program captured a range of detailed and interesting qualitative, quantitative and prospective data about young people's everyday mood, stresses, responses and general functioning.

\section{Using ketamine to model thought disorder in schizophrenia}

\section{S Rossell', V Curran², C Morgan² \\ 'Mental Health Research Institute, Melbourne, Australia; and 2University College London, London, United Kingdom}

Background: Ketamine is used acutely as a model of schizophrenia. It has been suggested that chronic ketamine use may also mimic aspects of this disorder, in particular cognitive function. Semantic processing deficits are considered to be central to cognitive impairments in schizophrenia and are related to thought disorder. This study aimed to characterize semantic impairments following both acute and chronic ketamine. Methods: We examined the acute effects of ketamine using a double-blind, placebo-controlled, independent groups design with 48 volunteers examining the effects of two doses of ketamine $(100 \mathrm{ng} / \mathrm{ml}$ and $200 \mathrm{ng} / \mathrm{ml})$. The chronic effects of ketamine were explored with 32 volunteers, 16 regular ketamine users and 16 matched polydrug controls. Semantic processing was examined using a lexical-decision semantic priming task with a frequency (high and low) and stimulus onset asynchrony (SOA; short $-200 \mathrm{~ms}$, long $-750 \mathrm{~ms}$ ) manipulation. Schizophrenic and dissociative symptoms were also examined.

Results: Acute ketamine produced a dose-dependent reduction in priming (hypopriming) and increased schizophrenic thought disorder. Ketamine users showed impaired priming for low-frequency words at the long SOA compared with polydrug controls, and there was some evidence of increased priming for high-frequency words. Ketamine users did not differ 